\title{
Evaluation and Modeling of Anisotropic Stress Effect on Hydrogen Diffusion in Bec Iron*1
}

\author{
Shuki Nagase ${ }^{1, * 2}$ and Ryosuke Matsumoto ${ }^{2, * 3}$ \\ ${ }^{1}$ Department of Mechanical Engineering and Science, Graduate School of Engineering, Kyoto University, Kyoto 615-8540, Japan \\ ${ }^{2}$ Nagamori Institute of Actuators, Kyoto University of Advanced Science, Kyoto 615-8577, Japan
}

\begin{abstract}
The diffusion behavior of interstitial hydrogen in steel should be clarified to reveal the mechanism of hydrogen embrittlement. In this study, we performed molecular dynamics (MD) simulations to elucidate the relationship between various stress conditions and the diffusion coefficients of interstitial hydrogen in body-centered-cubic (bcc) iron. The results reveal that the diffusion coefficients are independent of isotropic stress and exhibit strong anisotropic stress dependence under uniaxial stress along the $\langle 100\rangle$ direction. The stress-induced deformation of the atomic structure around the octahedral interstitial site (O site) was examined to elucidate the origin of the anisotropy of the stress dependence. Moreover, a model that predicts the activation enthalpy was derived by quantitatively evaluating the deformation around the $\mathrm{O}$ site. The activation enthalpy predicted by the model was consistent with the results of MD simulations when the deformation around the $\mathrm{O}$ site was not substantial. [doi:10.2320/matertrans.Z-M2020823]
\end{abstract}

(Received February 23, 2020; Accepted April 1, 2020; Published June 25, 2020)

Keywords: hydrogen, diffusion, molecular dynamics, stress effect, activation enthalpy

\section{Introduction}

Environmental issues such as global warming are attracting attention and are spurring interest in new energy alternatives to fossil fuels. Among them, hydrogen is expected to be used as an alternative energy because it generates only water, does not generate carbon dioxide, and has little environmental load in combustion, which is the most important reaction in energy utilization. ${ }^{1)}$

When hydrogen is used as a secondary energy source, it is typically stored at high pressures, and steel materials are expected to be used as structural members for hydrogen storage on the basis of cost. In a hydrogen environment, the strength of not only steels but also various metals is known to decrease because of hydrogen embrittlement; ${ }^{2,3)}$ however, the embrittlement mechanism is unclear. Clarifying the mechanisms of hydrogen embrittlement in steel materials requires knowledge of the exact distribution of hydrogen. Therefore, understanding the diffusion behavior of hydrogen has become a major issue.

Hydrogen diffusion in metals is described by the diffusion equation (eq. (1)) that includes the isotropic-stress-gradient term (second term on the right side): ${ }^{4-6)}$

$$
\boldsymbol{J}=-D \nabla c+\frac{D c V_{\mathrm{H}}}{R T} \nabla \sigma_{\mathrm{h}}
$$

where $J$ is the diffusion flux of hydrogen, $D$ is the diffusion coefficient, $c$ is the hydrogen concentration, $V_{\mathrm{H}}$ is the molar volume of hydrogen, $R$ is the gas constant, $T$ is temperature, and $\sigma_{\mathrm{h}}$ is the isotropic stress. Equation (1) shows that hydrogen transport occurs in the direction of higher isotropic stress. However, the dependence of diffusion coefficient $D$ in eq. (1) on the stress has not been sufficiently studied. Taketomi and Matsumoto have observed strong anisotropy in

\footnotetext{
${ }^{* 1}$ This Paper was Originally Published in J. Soc. Mater. Sci., Japan 69 (2020) 119-125. All captions of figures and tables were slightly modified. ${ }^{* 2}$ Graduate Student, Kyoto University

${ }^{* 3}$ Corresponding author, E-mail: matsumoto.ryosuke@kuas.ac.jp
}

the hydrogen diffusion behavior around dislocations in iron and have speculated that this anisotropy is caused by the contribution of anisotropic stress. ${ }^{7)}$ In addition, when the hydrogen microprint method is used for iron, silver particles precipitated at the position of hydrogen release have been reported to not be evenly distributed at grain boundaries or in grains. This uneven distribution may reflect the anisotropy of hydrogen diffusion due to residual stress per grain. 8,9 ) However, a detailed evaluation of the dependence on anisotropic stress and its reflection in the diffusion equation has not yet been reported.

Regarding the temperature dependence of the diffusion coefficient, quantum effects such as the tunneling effect are known to occur in the low-temperature region, and the diffusion mechanism changes remarkably. ${ }^{10)}$ However, the classical treatment is effective because hydrogen diffusion via the classical thermal activation process is dominant at temperatures higher than room temperature. The purpose of the present study is to analyze the diffusion behavior of hydrogen in iron by a molecular dynamics (MD) method and to clarify the stress dependence of the classical mechanical diffusion at temperature greater than room temperature, which is comparatively easy to handle. Furthermore, we construct a model that can explain the stress dependence of the activation enthalpy of diffusion by considering the stress-induced change in atomic configuration.

\section{Analysis Methods and Analysis Conditions}

\subsection{Overview}

To evaluate the effect of anisotropic stress on the hydrogen diffusion behavior in body-centered-cubic (bcc) iron, an MD method was used to simulate hydrogen atom diffusion. The time history of the coordinates of a hydrogen atom was obtained, and the mean square displacement (MSD) of a hydrogen atom was evaluated. In addition, the diffusion coefficient in the $\langle 100\rangle$ direction under each investigated temperature was calculated from the Einstein relation, and the activation enthalpy and frequency factor of the diffusion were 
obtained from the corresponding Arrhenius plot. This analysis was performed under various stress conditions to evaluate the stress dependence of the activation enthalpy of diffusion and the frequency factor.

\subsection{Analysis model and analysis conditions}

The [100], [010], and [001] directions of the bcc iron lattice were taken along the $x$-, $y$-, and $z$-axes, respectively. The initial position of hydrogen atoms was tetrahedral interstitial sites ( $\mathrm{T}$ sites) of the bcc iron lattice. The temperature was controlled by the velocity scaling method under 11 temperatures: 300, 330, 360, 400, 450, 500, 600, $700,800,900$, and $1000 \mathrm{~K}$. The stress conditions were isotropic stress $\sigma_{\mathrm{h}}\left(-3 \mathrm{GPa} \leq \sigma_{\mathrm{h}} \leq 3 \mathrm{GPa}\right)$, shear stress $\tau_{x y}$ $\left(0.2 \mathrm{GPa} \leq\left|\tau_{x y}\right| \leq 2 \mathrm{GPa}\right)$, and uniaxial stress in the $x$-axis direction $\sigma_{x}\left(-1.5 \mathrm{GPa} \leq \sigma_{x} \leq 1.5 \mathrm{GPa}\right)$. The positive signs of isotropic and uniaxial stresses indicate tension. In the uniaxial stress state, the cell size was controlled so that the stress in the two axes ( $y$-and $z$-axes) other than the stress loading direction ( $x$-axis) became 0 . When isotropic stress and uniaxial stress were applied, the number of atoms was 2000 iron and 1 hydrogen, totaling 2001. The cell dimensions under no stress were $2.87,2.87$, and $2.87 \mathrm{~nm}$ along the $x$-, $y$-, and $z$-axes, respectively. When a shear stress was applied, to give a shear stress $\tau_{x y}$ acting on a plane perpendicular to the $x$ - and $y$-axes, the [110], [110], and [001] directions of the bcc lattice were taken as the $x^{\prime}-, y^{\prime}$-, and $z^{\prime}$-axes, respectively. In this model, the normal stresses in the $x^{\prime}$ and $y^{\prime}$ directions $\left(\sigma_{x^{\prime}}=\tau\right.$ and $\left.\sigma_{y^{\prime}}=-\tau\right)$ are given to realize a pure shear stress state $\left(\tau_{x y}=-\tau\right)$. The total number of atoms was 1961, comprising 1960 iron atoms and 1 hydrogen atom. The cell dimensions under no stress were $2.84,2.84$, and $2.87 \mathrm{~nm}$ in the $x^{\prime}, y^{\prime}$, and $z^{\prime}$ directions, respectively.

In all analyses in the present study, periodic boundary conditions were applied in all directions. For the time integration method, the multiple time integration method by reversible reference system propagator algorithms $(\mathrm{rRESPA})^{11)}$ was used; the time steps were $\Delta t=2 \mathrm{fs}$ for iron atoms and $\Delta t / 24$ for the hydrogen atom, and the total calculation time was $3 \mathrm{~ns}$. For the interatomic potential, we used the EAM potential proposed by Wen et al. ${ }^{12)}$ Matsumoto et al. performed comparative calculations using this potential and first-principles calculations with respect to the heat of solution of hydrogen into the $\mathrm{T}$ site and $\mathrm{O}$ site of bcc iron. Their results showed that the heats of solution obtained by the two methods exhibited a similar strain dependence. ${ }^{13)}$ Therefore, this potential is suitable for the analysis of hydrogen diffusion behavior under stress.

\subsection{Method for evaluating diffusion coefficient and activation enthalpy of diffusion}

The diffusion coefficient of hydrogen atom $D$ in the $d$ dimension was obtained from the following Einstein relation on the basis of the time history of the atomic coordinates of hydrogen:

$$
D=\lim _{\Delta \tau \rightarrow \infty} \frac{1}{2 d \Delta \tau}\left\langle\left|\boldsymbol{r}\left(t_{0}+\Delta \tau\right)-\boldsymbol{r}\left(t_{0}\right)\right|^{2}\right\rangle
$$

where $\boldsymbol{r}$ is the position coordinate of the hydrogen atom and $\left\langle\left|\boldsymbol{r}\left(t_{0}+\Delta \tau\right)-\boldsymbol{r}\left(t_{0}\right)\right|^{2}\right\rangle$ is the MSD during $\Delta t(1 \mathrm{ps} \leq \Delta t \leq$
5 ps). From eq. (2), the diffusion coefficient can be obtained by dividing $2 d$ into the slope of the line fitted to the plot of MSD as a function of the time width of the MSD, $\Delta \tau$. The slope was determined using the least-squares method. When the MSD was calculated, the first $10 \mathrm{ps}$ in the time history data of the MD calculation was excluded as the relaxation time and the $\operatorname{MSD}(\Delta \tau)$ was derived from the average of the square displacements of various initial time $t_{0}$.

The activation enthalpy of diffusion $E_{\mathrm{a}}$ was obtained from the following Arrhenius equation:

$$
D=D_{0} \exp \left(-\frac{E_{\mathrm{a}}}{k_{\mathrm{B}} T}\right)
$$

where $D_{0}$ is the frequency factor, $k_{\mathrm{B}}$ is the Boltzmann constant, and $T$ is the temperature. According to eq. (3), the slope and the intercept of the Arrhenius plot, which displays the logarithm of $D(\ln D)$ plotted as a function of the reciprocal of the temperature $(1 / T)$, are $-E_{\mathrm{a}} / k_{\mathrm{B}}$ and $\ln D_{0}$, respectively. That is, the activation enthalpy $E_{\mathrm{a}}$ and frequency factor of diffusion $D_{0}$ can be obtained by linear approximation from the least-squares method to the Arrhenius plot of the diffusion coefficient obtained for each temperature condition. Furthermore, if $D$ is replaced with one-dimensional diffusion coefficients $D_{x}, D_{y}$, and $D_{z}$, where $d=1$ in eq. (2), the activation enthalpies of diffusion in the $x, y$, and $z$ directions are obtained; these enthalpies are expressed as $E_{\mathrm{a}_{-} x}$, $E_{\mathrm{a}_{-} y}$, and $E_{\mathrm{a}_{-} z}$, respectively.

\section{Analysis Results}

\subsection{Evaluation of diffusion coefficient under no stress}

Arrhenius plots of the diffusion coefficients obtained under no-stress conditions are shown in Fig. 1. As discussed in Section 2.3, the activation enthalpy $E_{\mathrm{a}}$ and frequency factor $D_{0}$ were obtained from the slope and the intercept of the approximate straight line. Here $E_{\mathrm{a}}=0.034 \mathrm{eV}$ and $D_{0}=$ $1.59 \times 10^{-8} \mathrm{~m}^{2} / \mathrm{s}$ were obtained.

\subsection{Evaluation of activation enthalpy of diffusion under stress}

The activation enthalpy of diffusion obtained under isotropic stress is shown in Fig. 2. The activation enthalpy was confirmed to be approximately the same value $(0.032-$

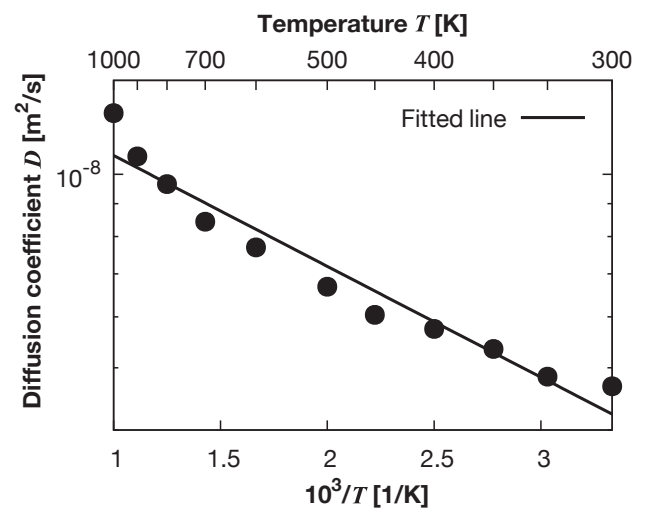

Fig. 1 Arrhenius plot of diffusion coefficients against temperature: The activation enthalpy $E_{\mathrm{a}}$ and frequency factor $D_{0}$ are obtained from the slope and intercept of the fitted line, respectively. 


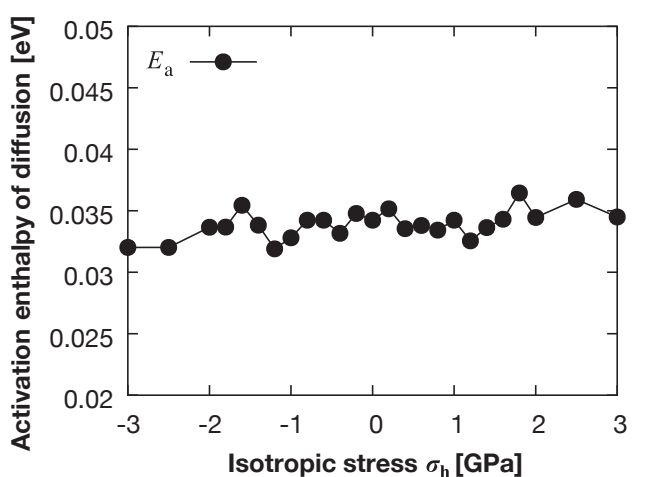

Fig. 2 Relationship between activation enthalpy of diffusion $E_{\mathrm{a}}$ and isotropic strain $\sigma_{\mathrm{h}}$.

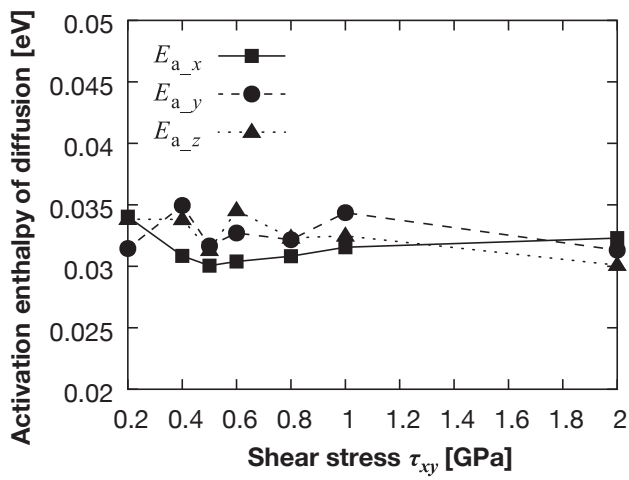

Fig. 3 Relationship between activation enthalpy of diffusion along the $x$-, $y$-, and $z$-axes $\left(E_{\mathrm{a}_{-} x}, E_{\mathrm{a}_{-} y}\right.$, and $\left.E_{\mathrm{a}_{-} z}\right)$ and shear stress $\tau_{x y}$.

$0.036 \mathrm{eV}$ ) irrespective of the magnitude of the isotropic stress.

The activation enthalpy of diffusion obtained under shear stress is shown in Fig. 3. Here, activation enthalpies $E_{\text {a }-x}$, $E_{\mathrm{a}_{-} y}$, and $E_{\mathrm{a}_{-} z}$ in the $x-, y$-, and $z$-axis directions introduced in Section 2.3 are shown. We confirmed that the activation enthalpy is approximately the same value $(0.030-0.035 \mathrm{eV})$ irrespective of the magnitude of stress. In addition, the activation enthalpies in the $x$ - and $y$-axis directions, where shear stress is applied, and in the $z$-axis direction, where shear stress is not applied, are also approximately the same. Thus, the activation enthalpy of hydrogen diffusion in bcc iron is not affected by either isotropic stress or shear stress along the $\{100\}$ plane.

The activation enthalpy of diffusion obtained under uniaxial stress is shown in Fig. 4. Because the activation enthalpies in the $y$ and $z$ directions perpendicular to the $x$-axis to which uniaxial stress is applied are equal as a consequence of symmetry, they are expressed as $E_{\mathrm{a} \perp}$ and are determined as the average of $E_{\mathrm{a}_{-} y}$ and $E_{\mathrm{a}_{-} z}$. The activation enthalpy in the direction parallel to the uniaxial stress $E_{\mathrm{a} / /}$ is defined using the activation enthalpy in the $x$-axis direction $E_{\mathrm{a}_{-} x}$. Figure 4 shows that, in the direction parallel to the uniaxial stress, the activation enthalpy $E_{\mathrm{a} / /}$ decreases with compression and increases with tension. By contrast, in the direction perpendicular to the uniaxial stress, the activation enthalpy $E_{\mathrm{a} \perp}$ increases under both compression and tension. As previously described, strong anisotropy is observed in the stress dependence of activation enthalpy of diffusion under uniaxial stress along $\langle 100\rangle$.

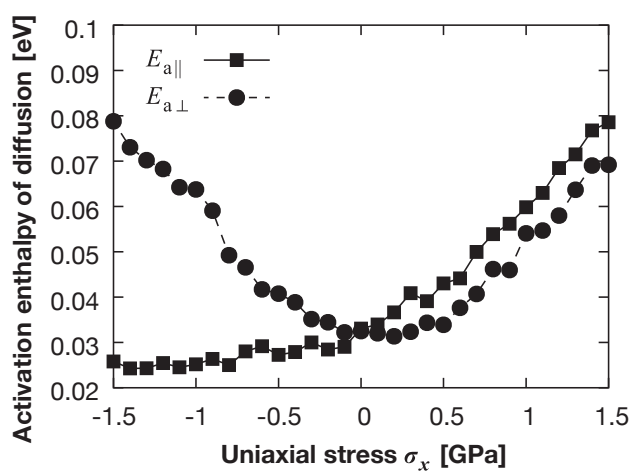

Fig. 4 Relationship between activation enthalpy of diffusion ( $E_{\mathrm{a} / /}$ and $\left.E_{\mathrm{a} \perp}\right)$ and uniaxial stress $\sigma_{x}: E_{\mathrm{a} / /}$ and $E_{\mathrm{a} \perp}$ are the activation enthalpy of diffusion parallel to uniaxial stress and that perpendicular to uniaxial stress, respectively.

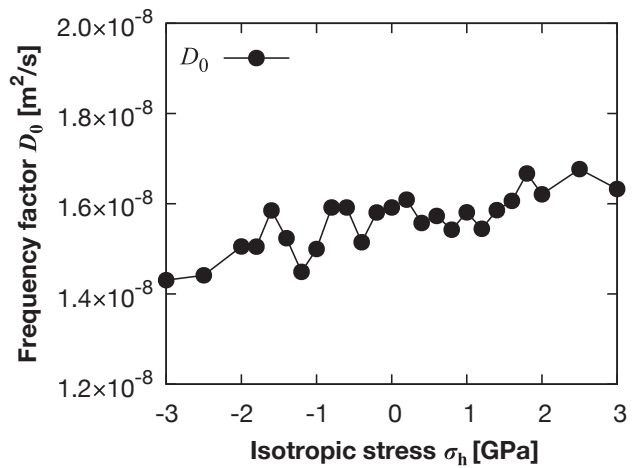

Fig. 5 Relationship between frequency factor $D_{0}$ and isotropic stress $\sigma_{\mathrm{h}}$.

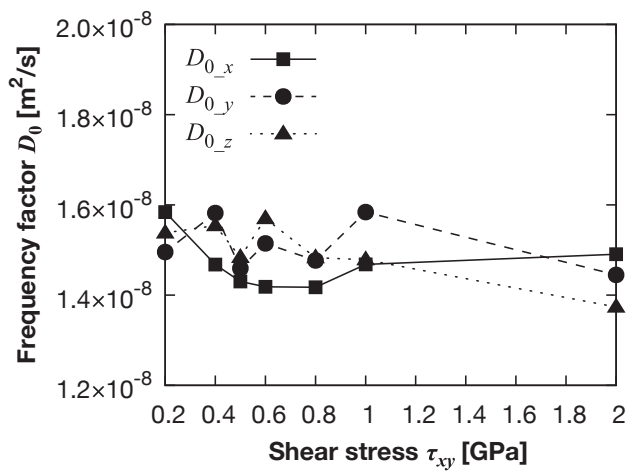

Fig. 6 Relationship between the frequency factor along each axis $\left(D_{0_{-} x}\right.$, $D_{0_{-} y}$, and $D_{0_{-} z}$ ) and shear stress $\tau_{x y}$.

\subsection{Frequency factors under stresses}

The frequency factors obtained under isotropic stress and shear stress are shown in Figs. 5 and 6, respectively. In both cases, the value increases and decreases slightly in response to stress but is clearly almost constant.

The frequency factor obtained under uniaxial stress is shown in Fig. 7. Here, as in the case of the activation enthalpy, the frequency factor in the direction parallel to the uniaxial stress $D_{0 / /}$ and the frequency factor in the vertical direction $D_{0 \perp}$ are defined. Although the frequency factor $D_{0 / /}$ is almost constant, the frequency factor $D_{0 \perp}$ increases in both compression and tension.

Ramasubramaniam et al. performed first-principles calculations showing that the energy barriers for diffusion and the 


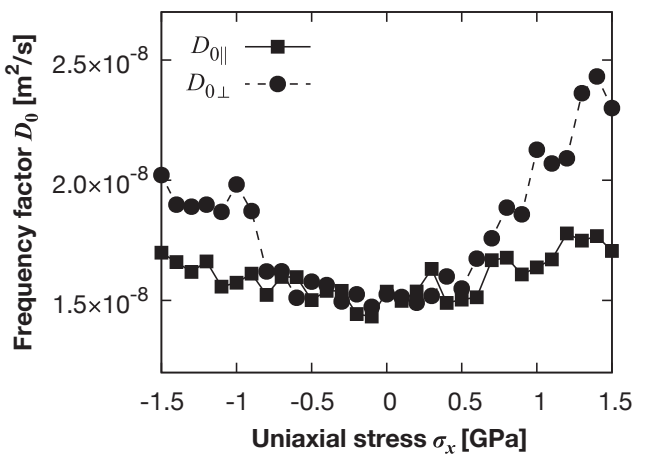

Fig. 7 Relationship between frequency factor $\left(D_{0 / /}\right.$ and $\left.D_{0 \perp}\right)$ and uniaxial stress $\sigma_{x}$.

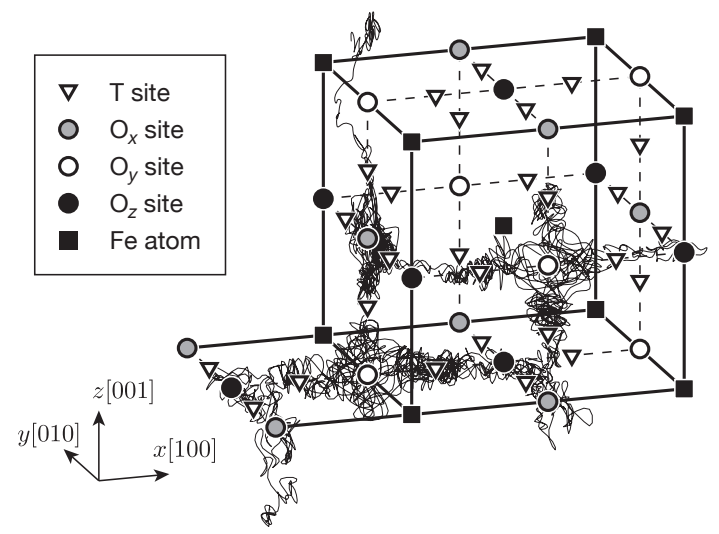

Fig. 8 Trajectory of hydrogen under the condition where the temperature is $300 \mathrm{~K}$ and the stress is $0 \mathrm{GPa}$ : The hydrogen atom vibrates at one T site and jumps to a neighboring $\mathrm{T}$ site via the neighboring $\mathrm{O}$ site between them.

frequency factor are independent of isotropic stress and shear stress and reported the uniaxial strain dependence. ${ }^{14)}$ Our previously described results are consistent with this trend.

\subsection{Diffusion path of hydrogen atom}

The bcc lattice includes three crystallographically distinct octahedral interstitial sites (O sites) that differ by the direction of the four-fold rotationally symmetric axis of the octahedron. Hereinafter, the sites whose axes of symmetry are parallel to the $x$ [100], $y$ [010], and $z$ [001] axes are referred to as $\mathrm{O}_{x}, \mathrm{O}_{y}$, and $\mathrm{O}_{z}$ sites, respectively.

Figure 8 shows the trajectory of hydrogen atoms for $11.5 \mathrm{ps}$ at $0 \mathrm{GPa}$ stress and at $300 \mathrm{~K}$. Hydrogen is vibrating at the $\mathrm{T}$ site and diffusing along the $\mathrm{O}$ site to another $\mathrm{T}$ site. In addition, hydrogen diffusing in the $x$-axis direction passes near $\mathrm{O}_{y}$ and $\mathrm{O}_{z}$ sites, hydrogen diffusing in the $y$-axis direction passes near $\mathrm{O}_{z}$ and $\mathrm{O}_{x}$ sites, and hydrogen diffusing in the $z$-axis direction passes near $\mathrm{O}_{x}$ and $\mathrm{O}_{y}$ sites.

\section{Discussion}

As evident from the results described in Section 3.2, compared with the frequency factor, the activation enthalpy changes remarkably with $\langle 100\rangle$ uniaxial stress. Therefore, in the following discussion, we focus on the activation enthalpy and discuss its stress dependence. As shown in Section 3.4, because hydrogen passes near the $\mathrm{O}$ site when it diffuses between two $\mathrm{T}$ sites, the activation enthalpy of diffusion is thought to depend approximately on the energy when hydrogen occupies the $\mathrm{T}$ site or the $\mathrm{O}$ site. Here, to consider the cause of stress dependence and anisotropy of the activation enthalpy shown in Section 3.2, we consider the stability of hydrogen at each site. The uniaxial straindependent anisotropy of the heat of solution is known to be negligible when hydrogen is inserted into the $\mathrm{T}$ site of bcc iron. ${ }^{13)}$ However, the heat of solution of hydrogen at the $\mathrm{O}$ site depends on the direction of uniaxial strain. ${ }^{13)}$ Thus, the anisotropy of the stress dependence of the activation enthalpy can be qualitatively explained from the effect of strain at the O site.

Compared with a regular octahedron, an octahedron surrounding the $\mathrm{O}$ site has a collapsed shape along one axis of symmetry. When the strain in comparison with that of a regular octahedron is expressed as the degree of anisotropy (DOA), a smaller DOA indicates lower energy at the O-site occupancy, and the energy difference between the $\mathrm{T}$ and $\mathrm{O}$ sites (i.e., the activation enthalpy) becomes smaller. Conversely, the greater the DOA, the greater the activation enthalpy. For example, when a uniaxial tensile strain of $0.5 \%$ is applied to reduce the DOA of the octahedron around the $\mathrm{O}$ site, the energy difference is $0.027 \mathrm{eV}$, which is smaller than the energy difference of $0.039 \mathrm{eV}$ in the unstrained state. However, when a uniaxial compressive strain of $0.5 \%$ was applied to increase the DOA, the energy difference was $0.053 \mathrm{eV}$, which is larger than that in the unstrained state.

On the basis of the previous results, we consider the activation enthalpy under uniaxial stress. First, when compressive stress is applied in the $x$-axis direction, the DOA of the $\mathrm{O}_{x}$ site increases and the DOA of the $\mathrm{O}_{y}$ and $\mathrm{O}_{z}$ sites decreases. As shown in Fig. 8, the hydrogen diffusion path in the $x$-axis direction (parallel to the stress) passes near the $\mathrm{O}_{y}$ and $\mathrm{O}_{z}$ sites, both of which have a small DOA; thus, the activation enthalpy decreases. This result matches that in Fig. 4. In the diffusion path along the $y$ - $[z-]$ axis direction (normal to the stress), the activation enthalpy is rate-limiting toward the larger path because it passes near the $\mathrm{O}_{x}$ site with the larger DOA and the $\mathrm{O}_{z}\left[\mathrm{O}_{y}\right]$ site with a smaller DOA. However, when tensile stress is applied in the $x$-axis direction, the DOA of the $\mathrm{O}_{x}$ site becomes small and the DOA of the $\mathrm{O}_{y}$ and $\mathrm{O}_{z}$ sites becomes large. The diffusion path in the $x$-axis direction (parallel to the stress) passes near $\mathrm{O}_{y}$ and $\mathrm{O}_{z}$ sites, both of which have a large DOA, and the diffusion path in the $y$ - [z-] axis direction (perpendicular to the stress) passes near $\mathrm{O}_{x}$ sites with a small DOA and $\mathrm{O}_{z}\left[\mathrm{O}_{y}\right]$ sites with a large DOA. That is, the activation enthalpy increases in all axis directions, which matches the results shown in Fig. 4.

\section{Modeling of Stress Dependence of Activation En- thalpy}

\subsection{Formula for predicting activation enthalpy}

For the activation enthalpy of diffusion under stress, the activation energy is corrected by $\left(\varepsilon^{*}{ }_{x x} \sigma_{x x}+\varepsilon^{*}{ }_{y y} \sigma_{y y}+\right.$ $\left.\varepsilon^{*}{ }_{z z} \sigma_{z z}\right) \times V$ using the axial strain of the calculation cell at saddle-point configuration $\left(\varepsilon^{*} x x, \varepsilon^{*}{ }_{y y}, \varepsilon_{z z}^{*}\right)$, the applied stress $\left(\sigma_{x x}, \sigma_{y y}, \sigma_{z z}\right)$, and the calculation cell volume $V$. In this 


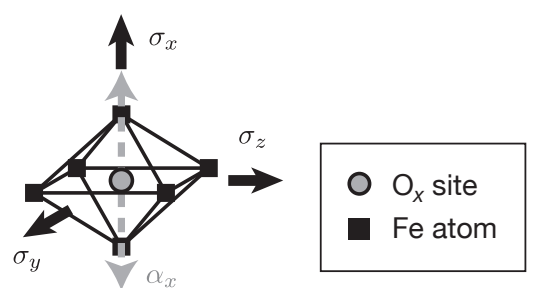

Fig. 9 Stress-induced deformation of the atomic structure around the $\mathrm{O}$ site.

method, the activation enthalpy cannot be expressed independently of the isotropic stress. Therefore, a formula is derived to predict the activation enthalpy of diffusion on the basis of the DOA of the octahedron surrounding the $\mathrm{O}$ site, as introduced in Section 4. First, we consider the index of the DOA quantitatively. In the case of the $\mathrm{O}_{x}$ site, when the longitudinal strain due to the stress in the $x$-axis direction and the lateral strain due to the stress in the $y$-and $z$-axis directions are considered, the following equation $\alpha_{x}$ is expressed as the DOA of the $\mathrm{O}_{x}$ site (Fig. 9):

$$
\alpha_{x}=\sigma_{x}-\frac{1}{2}\left(\sigma_{y}+\sigma_{z}\right)
$$

Similarly, the degrees of anisotropy of the $\mathrm{O}_{y}$ and $\mathrm{O}_{z}$ sites are represented by the expressions

$$
\begin{aligned}
& \alpha_{y}=\sigma_{y}-\frac{1}{2}\left(\sigma_{z}+\sigma_{x}\right) \\
& \alpha_{z}=\sigma_{z}-\frac{1}{2}\left(\sigma_{x}+\sigma_{y}\right)
\end{aligned}
$$

Because the diffusion path in the $x$-axis direction passes near $\mathrm{O}_{y}$ and $\mathrm{O}_{z}$ sites, the activation enthalpy becomes a function of their DOA $\alpha_{y}$ and $\alpha_{z}$, as shown in the following equation:

$$
E_{\mathrm{a}_{-} x}=F\left(\alpha_{y}, \alpha_{z}\right)
$$

Because diffusion is the same along all three axes, the same function $F$ is used to express $E_{\mathrm{a}_{-} y}$ and $E_{\mathrm{a}_{-} z}$ :

$$
\begin{aligned}
& E_{\mathrm{a}_{-} y}=F\left(\alpha_{z}, \alpha_{x}\right) \\
& E_{\mathrm{a}_{-} z}=F\left(\alpha_{x}, \alpha_{y}\right)
\end{aligned}
$$

Because $\alpha_{x}, \alpha_{y}$, and $\alpha_{z}$ always become 0 in the case of isotropic stress and because the term for shear stress is not included, the activation enthalpy does not depend on isotropic stress and shear stress.

When the symmetry of hydrogen diffusion is considered, $F(u, v)$ is symmetric about $u$ and $v$; thus, $F$ can be expressed by a polynomial in two variables:

$$
\begin{aligned}
F(u, v)= & a_{00}+a_{01}(u+v)+a_{02}\left(u^{2}+v^{2}\right)+a_{11} u v \\
& +a_{12}\left(u^{2} v+u v^{2}\right)+a_{22} u^{2} v^{2}
\end{aligned}
$$

The activation enthalpy obtained by MD calculation was plotted against the DOA $\alpha$ and its coefficient was determined by fitting eq. (10) (Fig. 10). When the fitted surface was obtained, in addition to the activation enthalpy for uniaxial stress $\sigma_{x}\left(-1.5 \mathrm{GPa} \leq \sigma_{x} \leq 1.5 \mathrm{GPa}\right)$ shown in Section 3.2, those for biaxial stresses in the $x$ and $y$ directions, $\sigma_{x}$ and $\sigma_{y}$ ( $\left.0 \mathrm{GPa} \leq \sigma_{x}, \sigma_{y} \leq 2 \mathrm{GPa}\right)$, were used. The analytical model and analytical conditions for biaxial stress conditions are similar to those for uniaxial stress conditions (Section 2.2).

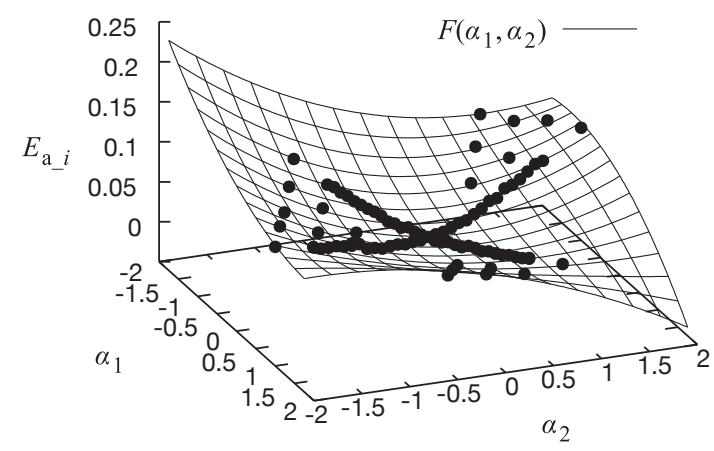

Fig. 10 Fitted results of $F\left(\alpha_{1}, \alpha_{2}\right)$ to the MD simulations.

Table 1 Fitted constants in $F\left(\alpha_{1}, \alpha_{2}\right)$.

\begin{tabular}{ccc}
\hline$a_{00}$ & $a_{01}$ & $a_{02}$ \\
$3.35 \times 10^{-2}$ & $-1.44 \times 10^{-2}$ & $1.73 \times 10^{-2}$ \\
\hline$a_{11}$ & $a_{12}$ & $a_{22}$ \\
$0.08 \times 10^{-2}$ & $-0.64 \times 10^{-2}$ & $-0.48 \times 10^{-2}$ \\
\hline
\end{tabular}

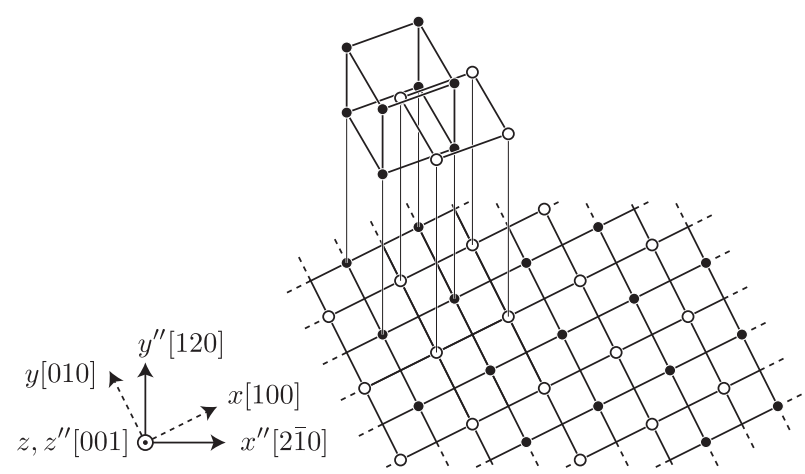

Fig. 11 Simulation model used to verify the accuracy of the prediction model.

The cell size was controlled such that the stress in the direction where no stress was applied became 0 . The obtained coefficients are shown in Table 1.

\subsection{Validating prediction formulas}

Equations (7)-(9) for predicting the activation enthalpy were verified under multiaxial stress conditions. To apply shear stress $\tau_{x y}$ in the directions of $x$ [100], $y$ [010], and $z$ [001], the [210], [120], and [001] directions were set to $x^{\prime \prime}$, $y^{\prime \prime}$, and $z^{\prime \prime}$ axes, respectively (Fig. 11), and normal stresses $\sigma_{x^{\prime \prime}}, \sigma_{y^{\prime \prime}}$, and $\sigma_{z^{\prime \prime}}$ were added. Thus, the normal stresses $\sigma_{x}, \sigma_{y}$, and $\sigma_{z}$ and shear stress $\tau_{x y}$ in the $x y z$ coordinate system become

$$
\begin{aligned}
& \left.\begin{array}{l}
\sigma_{x} \\
\sigma_{y}
\end{array}\right\}=\frac{1}{2}\left(\sigma_{x^{\prime \prime}}+\sigma_{y^{\prime \prime}}\right) \pm \frac{3}{5}\left(\frac{\sigma_{x^{\prime \prime}}-\sigma_{y^{\prime \prime}}}{2}\right) \\
& \sigma_{z}=\sigma_{z^{\prime \prime}} \\
& \tau_{x y}=-\frac{4}{5}\left(\frac{\sigma_{x^{\prime \prime}}-\sigma_{y^{\prime \prime}}}{2}\right)
\end{aligned}
$$

The analytical model comprises 2500 iron and 1 hydrogen atoms, for a total of 2501 atoms. The cell dimensions under 
Table 2 Comparison of the activation enthalpy obtained by MD simulations and by the prediction model.

\begin{tabular}{|c|c|c|c|c|c|c|c|c|c|c|}
\hline & \multicolumn{7}{|c|}{ Stress $(\mathrm{GPa})$} & \multicolumn{3}{|c|}{ Degree of anisotropy } \\
\hline & $\sigma_{x}$ & $\sigma_{y}$ & $\sigma_{z}$ & $\sigma_{x}$ & $\sigma_{y}$ & $\sigma_{z}$ & $\sigma_{\mathrm{eq}}$ & $\alpha_{x}$ & $\alpha_{y}$ & $\alpha_{z}$ \\
\hline (a) & -0.9 & -0.6 & -1 & -0.93 & -0.57 & -1 & 0.36 & -0.145 & 0.395 & -0.25 \\
\hline (b) & 1.5 & 1 & 1 & 1.55 & 0.95 & 1 & 0.50 & 0.575 & -0.325 & -0.25 \\
\hline (c) & -0.3 & 0.6 & 0.9 & -0.39 & 0.69 & 0.9 & 1.08 & -1.185 & 0.435 & 0.75 \\
\hline (d) & -0.4 & 0.8 & 0.5 & -0.52 & 0.92 & 0.5 & 1.08 & -1.23 & 0.93 & 0.3 \\
\hline (e) & 0.8 & -1 & -0.5 & 0.98 & -1.18 & -0.5 & 1.61 & 1.82 & -1.42 & -0.4 \\
\hline (f) & -1 & 1.5 & 1 & -1.25 & 1.75 & 1 & 2.29 & -2.625 & 1.875 & 0.75 \\
\hline (g) & 2 & -1 & 1 & 2.3 & -1.3 & 1 & 2.65 & 2.45 & -2.95 & 0.5 \\
\hline
\end{tabular}

\begin{tabular}{|c|c|c|c|c|c|c|c|c|c|}
\hline \multirow[b]{3}{*}{ (a) } & \multicolumn{6}{|c|}{ Activation enthalpy $E_{\mathrm{a}_{-} x}, E_{\mathrm{a} \_y}, E_{\mathrm{a}_{-} z}(\mathrm{eV})$} & \multicolumn{3}{|c|}{ Error $(\%)$} \\
\hline & \multicolumn{3}{|c|}{ MD simulations } & \multicolumn{3}{|c|}{ Prediction } & \multirow[b]{2}{*}{3} & \multirow[b]{2}{*}{7} & \multirow[b]{2}{*}{5} \\
\hline & 0.034 & 0.038 & 0.031 & 0.035 & 0.041 & 0.033 & & & \\
\hline (b) & 0.037 & 0.035 & 0.032 & 0.045 & 0.036 & 0.036 & 22 & 2 & 16 \\
\hline (c) & 0.037 & 0.046 & 0.049 & 0.027 & 0.067 & 0.068 & -29 & 44 & 37 \\
\hline (d) & 0.029 & 0.042 & 0.037 & 0.030 & 0.072 & 0.070 & 5 & 71 & 86 \\
\hline (e) & 0.043 & 0.041 & 0.032 & 0.103 & 0.077 & 0.092 & 138 & 85 & 185 \\
\hline (f) & 0.030 & 0.064 & 0.060 & 0.034 & 0.146 & 0.080 & 13 & 128 & 32 \\
\hline (g) & 0.061 & 0.027 & 0.055 & 0.189 & 0.070 & 0.013 & 208 & 158 & -76 \\
\hline
\end{tabular}

no stress are $3.21 \mathrm{~nm}, 3.21 \mathrm{~nm}$, and $2.87 \mathrm{~nm}$ on the $x^{\prime \prime}, y^{\prime \prime}$ and $z^{\prime \prime}$ axes, respectively.

Table 2 shows the verified stress conditions, the activation enthalpy obtained by MD calculation, the activation enthalpy obtained by prediction formula (10), and the error of the prediction formula relative to the MD calculation. They also show the DOA under each stress condition. Under conditions (a) and (b), where the DOA is small, the activation enthalpy is determined with high accuracy by the prediction formula; however, under conditions (e), (f), and (g), where the DOA is far from 0 , such that the equivalent stress $\sigma_{\text {eq }}$ exceeds 1.5 GPa, a substantial difference is observed between the activation enthalpy obtained by MD calculation and that obtained by prediction formula (10). Because the stress condition of the MD calculation used for fitting the prediction formula is not satisfied in this case, the error of the prediction formula is increased. Such stress conditions occur in very limited cases, such as in the immediate vicinity of dislocations. The accuracy is improved by adopting the sample data of the fitting more widely when greater accuracy is necessary for such stress conditions.

\section{Conclusion}

The following are the findings from MD simulations of hydrogen diffusion in bcc iron by determining the diffusion coefficients in the [100], [010], and [001] directions and evaluating the stress dependence of the diffusion coefficients.
- The value of the diffusion coefficient under isotropic stress and $\{100\}$ plane shear stress is almost constant, irrespective of the stress.

- Diffusion coefficients under $\langle 100\rangle$ uniaxial stress show different stress dependence in the directions parallel and perpendicular to the stress.

In addition, regarding the stress dependence of the obtained diffusion coefficient, we examined the cause of the stress dependence of the activation enthalpy of diffusion by analyzing the effect of stress on the deformation of the octahedron composed of neighboring atoms around the $\mathrm{O}$ site in bec iron. We found that anisotropy occurs in the stress dependence when the diffusion path passes near $\mathrm{O}$ sites with different deformation depending on the direction of diffusion. Furthermore, by quantitatively expressing the deformation of the octahedron around the $\mathrm{O}$ site, we developed a formula to predict the activation enthalpy and subsequently verified its accuracy. When the deformation of the $\mathrm{O}$ site is not large, the prediction of our formula agrees with the result of MD calculations with high accuracy, and its effectiveness can be shown.

\section{REFERENCES}

1) Y. Murakami: Int. J. Fract. 138 (2006) 167-195.

2) M. Nagumo: Fundamentals of Hydrogen Embrittlement, (Springer, Berlin, 2016) pp. 103-133.

3) G. Han, J. He, S. Fukuyama and K. Yokogawa: Acta Mater. 46 (1998) 4559-4570.

4) P. Sofronis and R.M. McMeeking: J. Mechan. Phys. Solids 37 (1989) 
317-350.

5) H. Kotake, R. Matsumoto, S. Taketomi and N. Miyazaki: Int. J. Press. Vessels Piping 85 (2008) 540-549.

6) T. Takayama, R. Matsumoto, S. Taketomi and N. Miyazaki: Int. J. Hydrogen Energ. 36 (2011) 1037-1045.

7) S. Taketomi, R. Matsumoto and N. Miyazaki: Acta Mater. 56 (2008) 3761-3769.

8) N. Ishikawa, H. Sueyoshi and A. Nagao: ISIJ Int. 56 (2016) 413-417.

9) M. Koyama, D. Yamasaki, T. Nagashima, C.C. Tasan and K. Tsuzaki: Scr. Mater. 129 (2017) 48-51.
10) H. Kimizuka, H. Mori and S. Ogata: Phys. Rev. B 83 (2011) 094110

11) M. Tuckerman, B.J. Berne and G.J. Martyna: J. Chem. Phys. 97 (1992) 1990-2001.

12) M. Wen, X. Xu, S. Fukuyama and K. Yokogawa: J. Mater. Res. 16 (2001) 3496-3502.

13) R. Matsumoto, Y. Inoue, S. Taketomi and N. Miyazaki: Scr. Mater. 60 (2009) 555-558.

14) A. Ramasubramaniam, M. Itakura, M. Ortiz and E.A. Carter: J. Mater. Res. 23 (2008) 2757-2773. 